\title{
EXACT SOLUTION FOR LARGE AMPLITUDE FLEXURAL VIBRATION OF NANOBEAMS USING NONLOCAL EULER-BERNOULLI THEORY
}

\author{
REZA NAZEMNEZHAD \\ School of Engineering, Damghan University, Damghan, Islamic Republic of Iran \\ e-mail: rnazemnezhad@du.ac.ir \\ SHAHROKH Hosseini-HASHEMI \\ School of Mechanical Engineering, Iran University of Science and Technology, Narmak, Tehran, Iran and \\ Center of Excellence in Railway Transportation, Iran University of Science and Technology, Narmak, Tehran, Iran
}

\begin{abstract}
In this paper, nonlinear free vibration of nanobeams with various end conditions is studied using the nonlocal elasticity within the frame work of Euler-Bernoulli theory with von Kármán nonlinearity. The equation of motion is obtained and the exact solution is established using elliptic integrals. Two comparison studies are carried out to demonstrate accuracy and applicability of the elliptic integrals method for nonlocal nonlinear free vibration analysis of nanobeams. It is observed that the phase plane diagrams of nanobeams in the presence of the small scale effect are symmetric ellipses, and consideration the small scale effect decreases the area of the diagram.
\end{abstract}

Keywords: nonlinear free vibration, nonlocal elasticity, nanobeam, exact solution, elliptic integrals

\section{Introduction}

Due to superior properties, nanostructures have attracted much attention in the recent years. Multiple recent experimental results have shown that as the size of the structures reduces to micro/nanoscale, the influences of atomic forces and small scale play a significant role in mechanical properties of these nano-structures (Chong et al., 2001; Fleck et al., 1994; Ma and Clarke, 1995). Thus, neglecting these effects in some cases may results in completely incorrect solutions and hence wrong designs. The classical continuum theories do not include any internal length scale. Consequently, these theories are expected to fail when the size of the structure becomes comparable with the internal length scale. Eringen's nonlocal theory is one of the well-known continuum mechanics theories (Eringen, 2002) that includes small scale effects with good accuracy to micro/nanoscale devises modeling. The nonlocal elasticity theory assumes that the stress at a point is a function of the strain at all neighbor points of the body.

In the recent years, studies of nanostructures by using the nonlocal elasticity theory have been an area of active research. Based on this theory, Reddy (2007) derived the equation of motion of various kinds of beam theories available (Euler-Bernoulli, Timoshenko, Reddy and Levinson) and reached analytical and numerical solutions on static deflections, buckling loads, and natural frequencies. Hosseini-Hashemi et al. (2013) considered surface effects on free vibration of EulerBernoulli and Timoshenko nanobeams by using the nonlocal elasticity theory. Xie et al. (2006) considered the radial buckling pressure of a simply supported multi-walled carbon nanotube in the presence of the small scale effects. Kiani (2010) studied free longitudinal vibration of tapered nanowires. In (Heireche et al., 2008; Narendar and Gopalakrishnan, 2009; Wang and $\mathrm{Hu}, 2005$; Wang, 2005; Wang and Varadan, 2007), the effect of small scales on wave propagation of single- and multi-walled carbon nanotubes was considered. As an application of Eringen's 
nonlocal theory to post-buckling analysis of single-walled carbon nanotubes, it can be referred to the work done by Setoodeh et al. (2011).

The nonlocal elasticity theory has also been used for nonlinear analyses of nanostructures, especially nanobeams. In this area, Reddy (2010) derived a nonlocal nonlinear formulation for bending of classical and shear deformation theories of beams and plates, but he did not present any numerical results. Yang and Lim (2009) examined the effect of the nonlocal parameter on nonlinear vibrations of Euler nanobeams. To evaluate the nonlinear natural frequencies, the method of multiple scales was applied to the governing equation. The method of multiple scales was also used in (Nazemnezhad and Hosseini-Hashemi, 2014; Hosseini-Hashemi et al., 2014) for evaluating the nonlocal nonlinear natural frequencies of functionally graded Euler-Bernoulli nanobeams. Fang et al. (2013), Ghorbanpour Arani et al. (2012), Ansari and Ramezannezhad (2011), Yang et al. (2010) and Ke et al. (2009) investigated nonlinear free vibration of single and multi-walled carbon nanotubes with the aid of the nonlocal elasticity theory.

In the mentioned references, a numerical method, the DQ method, semi analytical methods, the harmonic balance method and the method of multiple scales have been employed to solve the nonlocal nonlinear governing equations. Hence, no exact closed-form solution is available in the literature for nonlocal nonlinear analyses of nanobeams. Motivated by the literature survey, this study aims to investigate the nonlocal nonlinear free vibration of Euler-Bernoulli nanobeams by an exact solution called the elliptic integrals. To this end, firstly, the governing equation of motion of the Euler-Bernoulli nanobeam has been obtained in the nonlocal form. Then, a closed-form expression for the nonlocal nonlinear natural frequencies has been presented by using the elliptic integrals. Finally, the accuracy of the present exact results are compared with those reported in literature, and the small scale effects on the phase plane diagram and higher frequency ratios are considered in the results and discussion śection.

\section{Formulation}

Consider a nanobeam with length $L(0 \leqslant x \leqslant L)$, thickness $2 h(-h \leqslant z \leqslant h)$ and width $b$ $(-b / 2 \leqslant y \leqslant b / 2)$ (Fig. 1). Upon the Euler-Bernoulli beam model, the displacement field at any point of the nanobeam can be written as

$$
u_{x}(x, z, t)=U(x, t)-z \frac{\partial W(x, t)}{\partial x} \quad u_{z}(x, z, t)=W(x, t)
$$

where $U(x, t)$ and $W(x, t)$ are the displacement components of the mid-plane at time $t$. In accordance, the von Kármán type of the nonlinear strain-displacement relationship is

$$
\varepsilon_{x x}=\frac{\partial u_{x}}{\partial x}+\frac{1}{2}\left(\frac{\partial u_{z}}{\partial x}\right)^{2}=\frac{\partial U}{\partial x}-z \frac{\partial^{2} W}{\partial x^{2}}+\frac{1}{2}\left(\frac{\partial W}{\partial x}\right)^{2}
$$
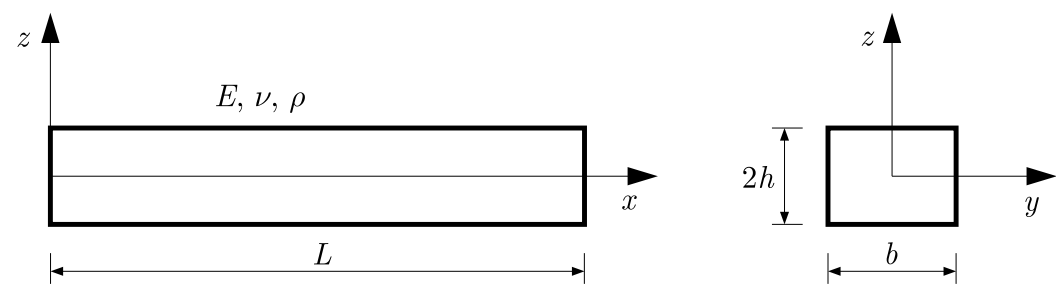

Fig. 1. Geometry of the problem

Now, by using Hamilton's principle, the nonlinear equations of motion of the nanobeam can be derived as

$$
\frac{\partial N_{x x}}{\partial x}=\rho A \frac{\partial^{2} U}{\partial t^{2}} \quad \frac{\partial^{2} M_{x x}}{\partial x^{2}}+\frac{\partial}{\partial x}\left(N_{x x} \frac{\partial W}{\partial x}\right)=\rho A \frac{\partial^{2} W}{\partial t^{2}}
$$


where $N_{x x}$ and $M_{x x}$ are the local force and bending moment resultants, respectively, given by

$$
N_{x x}=\int_{A} \sigma_{x x} d A=E A\left[\frac{\partial U}{\partial x}+\frac{1}{2}\left(\frac{\partial W}{\partial x}\right)^{2}\right] \quad M_{x x}=\int_{A} z \sigma_{x x} d A=-E I \frac{\partial^{2} W}{\partial x^{2}}
$$

Equations of motions, Eqs. (2.3), can be used in the nonlocal form

$$
\frac{\partial N_{x x}^{n l}}{\partial x}=\rho A \frac{\partial^{2} U}{\partial t^{2}} \quad \frac{\partial^{2} M_{x x}^{n l}}{\partial x^{2}}+\frac{\partial}{\partial x}\left(N_{x x}^{n l} \frac{\partial W}{\partial x}\right)=\rho A \frac{\partial^{2} W}{\partial t^{2}}
$$

where the superscript $n l$ denotes nonlocal. If the axial inertia is neglected, Eq. $(2.5)_{1}$ gives

$$
N_{x x}^{n l}=N_{0}=\text { const }
$$

The nonlocal force and bending moment resultants can be obtained by multiplying the left-hand side of Eqs. (2.4) by $\left(1-\mu \nabla^{2}\right)$, using Eqs. (2.5) and (2.6), and doing some mathematical manipulation. Then they are given by

$$
N_{x x}^{n l}=E A\left[\frac{\partial U}{\partial x}+\frac{1}{2}\left(\frac{\partial W}{\partial x}\right)^{2}\right] \quad M_{x x}^{n l}=-E I \frac{\partial^{2} W}{\partial x^{2}}+\mu\left(-N_{x x}^{n l} \frac{\partial^{2} W}{\partial x^{2}}+\rho A \frac{\partial^{2} W}{\partial t^{2}}\right)
$$

For nanobeams with immovable ends (i.e. $U$ and $W=0$, at $x=0$ and $L$ ) and with Eq. (2.6) in mind, integrating Eq. $(2.7)_{1}$ with respect to $x$ leads to

$$
N_{x x}^{n l}=N_{0}^{n l}=\frac{E A}{2 L} \int_{0}^{L}\left(\frac{\partial W}{\partial x}\right)^{2} d x
$$

Finally, by substituting Eqs. $(2.7)_{2}$ and (2.8) in Eq. $(2.5)_{2}$, the nonlocal nonlinear governing equation for the Euler-Bernoulli nanobeam can be obtained as

$$
-E I \frac{\partial^{4} W}{\partial x^{4}}+\mu \rho A \frac{\partial^{4} W}{\partial x^{2} \partial t^{2}}+P \int_{0}^{L}\left(\frac{\partial W}{\partial x}\right)^{2} d x\left(\frac{\partial^{2} W}{\partial x^{2}}-\mu \frac{\partial^{4} W}{\partial x^{4}}\right)-\rho A \frac{\partial^{2} W}{\partial t^{2}}=0
$$

where $P=E A /(2 L), I=2 b h^{3} / 3$, and the equation of motion of the conventional Euler-Bernoulli beam theory can be obtained from Eq. (2.9) by setting $\mu=0$.

For nonlinear free vibration analysis, the averaging technique over the space variable (Galerkin's method) is used to convert Eq. (2.9) into an ordinary differential equation. Hence, the transverse displacement of the $n$-th mode can be assumed as (Azrar et al., 1999, 2002)

$$
W(x, t)=D \phi(x) q(t)
$$

where $D$ is an arbitrary constant which represents the amplitude of deflection, $q(t)$ is a time dependent function to be determined and $\phi(x)$ is the linear mode shape obtained as follows (Rao, 2007)

$$
\begin{array}{ll}
\text { SS : } \quad \phi_{n}(x)=\sin \left(\frac{n \pi}{L} x\right) \\
\text { SC : } \quad \phi_{n}(x)=\sin \left(\zeta_{1} x\right)-\frac{\sin \left(\zeta_{1} L\right)}{\sinh \left(\zeta_{2} L\right)} \sinh \left(\zeta_{2} x\right)
\end{array}
$$

where

$$
\zeta_{1,2}=\sqrt{\frac{1}{2}\left( \pm \frac{\mu \rho A \omega^{2}}{E I}+\sqrt{\left.\left(\frac{\mu \rho A \omega^{2}}{E I}\right)^{2}+\frac{4 \rho A \omega^{2}}{E I}\right)}\right.}
$$


The parameters $\zeta_{1}$ and $\zeta_{2}$ can be determined from the eigenvalue equation

$$
\zeta_{2} \tan \left(\zeta_{1} L\right)-\zeta_{1} \tanh (\zeta 2 L)=0
$$

Substituting Eq. (2.10) and its derivatives into Eq. (2.9), then multiplying by the linear mode shape and integrating along the length of nanobeam, results in

$$
\ddot{q}-\gamma_{1} q+\gamma_{2} q^{3}=0
$$

where

$$
\begin{aligned}
\gamma_{1} & =\frac{E I a_{4}}{\mu \rho A a_{3}-\rho A a_{1}} & \gamma_{2} & =\frac{I P a_{2}\left(a_{3}-\mu a_{4}\right)}{\mu \rho A^{2} a_{3}-\rho A^{2} a_{1}}\left(\frac{D}{r}\right)^{2} \\
a_{1} & =\int_{0}^{L} \phi^{2}(x) d x & a_{2} & =\int_{0}^{L}\left(\frac{d \phi(x)}{d x}\right)^{2} d x \\
a_{3} & =\int_{0}^{L} \phi(x) \frac{d^{2} \phi(x)}{d x^{2}} d x & a_{4} & =\int_{0}^{L} \phi(x) \frac{d^{4} \phi(x)}{d x^{4}} d x
\end{aligned}
$$

and $r=\sqrt{I / A}$ is the radius of gyration.

Multiplying Eq. (2.12) by $\dot{q}$ and then integrating with respect to time, with the initial conditions $q(0)=1$ and $\dot{q}(0)=0$, leads to

$$
\left(\frac{d q}{d t}\right)^{2}=-\gamma_{1}\left(1-q^{2}\right)+\frac{\gamma_{2}}{2}\left(1-q^{4}\right)
$$

Equation (2.13) can be rewritten as

$$
\left(\frac{d q}{d(p t)}\right)^{2}=\left(1-q^{2}\right)\left(k^{2} q^{2}-k^{2}+1\right)
$$

where $p^{2}=-\gamma_{1}+\gamma_{2}$ and $k^{2}=\gamma_{2} /\left(2 p^{2}\right)$. Supposing $q=\cos \theta$, and complement the modulus as $p t=\int_{0}^{\theta}\left(1-k^{2} \sin ^{2} \theta\right)^{-1 / 2} d \theta$, the solution to Eq. (2.14) can be given by (Lestari and Hanagud, 2001)

$$
q(t)=c n[p t, k]
$$

where $c n$ is a Jacobi elliptic function. The Jacobi elliptic functions are real for real $p$ and real $k^{2}$ between 0 and 1 (Byrd et al., 1971). $K$ is defined by using the complete elliptic integral as

$$
K=\int_{0}^{\pi / 2} \frac{d \theta^{\prime}}{\sqrt{1-k^{2} \sin ^{2} \theta^{\prime}}}
$$

Therefore, the nonlocal natural frequency for nonlinear free vibration of nanoscale beams for each mode can be expressed as

$$
\omega_{n l}^{n l}=\frac{\pi \sqrt{-\gamma_{1}+\gamma_{2}}}{2 K}
$$

where the subscript $n l$ denotes nonlinear. 


\section{Results and discussion}

Before presenting numerical results, it is worth to note here that in order to convert the governing equation of motion, Eq. (2.9), into an ordinary differential equation, Eq. (2.12), single-mode Galerkin's method is used. This method can provide the nonlinear natural frequency of nanobeams with a good accuracy at low amplitude ratios (Ke et al., 2010). At higher amplitude ratios, multi-mode Galerkin's method is proposed because the effect of coupling of modes increases (Chandra and Raju, 1975). Therefore, in order to have the highest possible accuracy in the following discussion all results are presented for low amplitude ratios, $D / r \leqslant 3$.

Table 1 gives the fundamental local nonlinear frequency to fundamental local linear frequency ratio $\omega_{n l} / \omega_{l}$ of a nanobeam with SS and SC end conditions for various values of the amplitude ratio $D / r$. The solutions obtained by using numerical and approximate methods like GFEM (Galerkin Finite Element Method) (Bhashyam and Prathap, 1980), RGFEM (Reduced Galerkin Finite Element Method) (Bhashyam and Prathap, 1980), ASM (Assumed Space Method) (Evensen, 1968), and DQM (Differential Quadrature Method) (Malekzadeh and Shojaee, 2013) are also provided for direct comparison. It is observed that the present results agree very well with those given by Malekzadeh and Shojaee (2013), Bhashyam and Prathap (1980) and Evensen (1968). It is also seen from Table 1 that the present results obtained by the exact method are smaller than those given by numerical and approximate methods. It can be attributed to this fact that natural frequencies obtained by numerical and approximate methods are the upper bound of the exact ones.

Table 1. Comparison of the fundamental local frequency ratio $\omega_{n l} / \omega_{l}$ for a nanobeam with SS and SC end conditions $\mu=0, E=70 \mathrm{GPa}, \rho=2700 \mathrm{~kg} / \mathrm{m}^{3}, \nu=0.3, L / h=100$

\begin{tabular}{|c|c|c|c|c|c|c|c|c|c|}
\hline \multirow{2}{*}{$D / r$} & \multicolumn{4}{|c|}{ SS } & \multicolumn{5}{c|}{ SC } \\
\cline { 2 - 11 } & $\begin{array}{c}\text { Present } \\
\text { Exact) }\end{array}$ & $\begin{array}{c}\text { GFEM } \\
{[4]}\end{array}$ & $\begin{array}{c}\text { RGFEM } \\
{[4]}\end{array}$ & $\begin{array}{c}\text { ASM } \\
{[9]}\end{array}$ & $\begin{array}{c}\text { Present } \\
\text { (Exact) }\end{array}$ & $\begin{array}{c}\text { GFEM } \\
{[4]}\end{array}$ & $\begin{array}{c}\text { RGFEM } \\
{[4]}\end{array}$ & $\begin{array}{c}\text { ASM } \\
{[9]}\end{array}$ & $\begin{array}{c}\text { DQM } \\
{[21]}\end{array}$ \\
\hline \hline 0.5 & 1.0231 & 1.0308 & 1.0308 & 1.0308 & 1.0130 & 1.0165 & 1.0165 & 1.0676 & - \\
\hline 1.0 & 1.0892 & 1.1180 & 1.1180 & 1.1180 & 1.0507 & 1.0641 & 1.0641 & 1.0763 & 1.0641 \\
\hline 1.5 & 1.1902 & 1.2499 & 1.2499 & 1.2500 & 1.1105 & 1.1379 & 1.1379 & 1.0905 & - \\
\hline 2.0 & 1.3178 & 1.4142 & 1.4142 & 1.4142 & 1.1886 & 1.2319 & 1.2319 & 1.1099 & 1.2319 \\
\hline 2.5 & 1.4647 & 1.6008 & 1.6008 & 1.6008 & 1.2814 & 1.3407 & 1.3407 & 1.1342 & - \\
\hline 3.0 & 1.6257 & 1.8028 & 1.8028 & 1.8028 & 1.3860 & 1.4601 & 1.4601 & 1.1631 & 1.4605 \\
\hline 3.5 & 1.7970 & 2.0158 & 2.0158 & 2.0156 & 1.4998 & 1.5865 & 1.5865 & 1.1963 & - \\
\hline 4.0 & 1.9760 & 2.2365 & 2.2365 & 2.2361 & 1.6209 & 1.7166 & 1.7166 & 1.2334 & - \\
\hline 4.5 & 2.1608 & 2.4630 & 2.4630 & 2.4622 & 1.7477 & 1.8455 & 1.8455 & 1.2741 & - \\
\hline 5.0 & 2.3501 & 2.6937 & 2.6937 & 2.6926 & 1.8792 & 1.9651 & 1.9651 & 1.3181 & - \\
\hline
\end{tabular}

[4] - Bhashyam and Prathap (1980), [9] - Evensen (1968),

[21] - Malekzadeh and Shojaee (2013)

In Table 2, the fundamental nonlocal nonlinear frequency to fundamental local nonlinear frequency ratios $\omega_{n l}^{n l} / \omega_{n l}$ obtained by the present exact solution for a nanobeam with SS and SC boundary conditions is compared with those given by the semi-analytical approach called the multiple scales method (MSM) (Nazemnezhad and Hosseini-Hashemi, 2014). The results are listed for various values of the nanobeam length, amplitude ratio, and nonlocal parameter. Table 2 shows that the present exact results are identical to those reported by Nazemnezhad and Hosseini-Hashemi (2014) using the multiple scales method. However, the difference between the two approaches increases for higher amplitude ratios. This is due to the fact that the accuracy of the multiple scales method depends on the number of slow scale variables in the expansion series. 
Table 2. Comparison of the fundamental nonlocal frequency ratio $\omega_{n l}^{n l} / \omega_{n l}$ for a nanobeam with SS and SC end conditions $E=210 \mathrm{GPa}, \rho=2370 \mathrm{~kg} / \mathrm{m}^{3}, \nu=0.24, b=h=0.1 L$

\begin{tabular}{|c|c|c|c|c|c|c|c|c|}
\hline \multirow[b]{2}{*}{$\begin{array}{l}\text { Boundary } \\
\text { condition }\end{array}$} & \multirow[b]{2}{*}{$\begin{array}{c}\text { Length } \\
{[\mathrm{nm}]}\end{array}$} & \multirow[b]{2}{*}{$\begin{array}{c}\mu \\
{\left[\mathrm{nm}^{2}\right]}\end{array}$} & \multicolumn{3}{|c|}{$D / r=1$} & \multicolumn{3}{|c|}{$D / r=2$} \\
\hline & & & $\begin{array}{l}\text { Present } \\
\text { (Exact) }\end{array}$ & $\begin{array}{c}\text { MSM } \\
{[23]}\end{array}$ & $A D^{*} \cdot 100$ & $\begin{array}{l}\text { Present } \\
\text { (Exact) }\end{array}$ & $\begin{array}{c}\text { MSM } \\
{[23]}\end{array}$ & $A D \cdot 100$ \\
\hline \multirow{6}{*}{ SS } & \multirow{2}{*}{10} & 2 & 0.9278 & 0.9293 & 0.15 & 0.9509 & 0.9631 & 1.22 \\
\hline & & 4 & 0.8724 & 0.8754 & 0.30 & 0.9141 & 0.9379 & 2.38 \\
\hline & \multirow{2}{*}{20} & 2 & 0.9800 & 0.9803 & 0.03 & 0.9863 & 0.9893 & 0.30 \\
\hline & & 4 & 0.9613 & 0.9621 & 0.08 & 0.9736 & 0.9797 & 0.61 \\
\hline & \multirow{2}{*}{30} & 2 & 0.9909 & 0.9911 & 0.02 & 0.9938 & 0.9951 & 0.13 \\
\hline & & 4 & 0.9821 & 0.9824 & 0.03 & 0.9877 & 0.9905 & 0.28 \\
\hline \multirow{6}{*}{$\mathrm{SC}$} & \multirow{2}{*}{10} & 2 & 0.9189 & 0.9202 & 0.13 & 0.9547 & 0.9676 & 1.29 \\
\hline & & 4 & 0.8596 & 0.8625 & 0.29 & 0.9239 & 0.9519 & 2.80 \\
\hline & \multirow{2}{*}{20} & 2 & 0.9771 & 0.9774 & 0.03 & 0.9869 & 0.9899 & 0.30 \\
\hline & & 4 & 0.9561 & 0.9567 & 0.06 & 0.9751 & 0.9812 & 0.61 \\
\hline & \multirow{2}{*}{30} & 2 & 0.9896 & 0.9897 & 0.01 & 0.9940 & 0.9953 & 0.13 \\
\hline & & 4 & 0.9795 & 0.9798 & 0.03 & 0.9883 & 0.9909 & 0.26 \\
\hline
\end{tabular}

* - Absolute of difference, [23] - Nazemnezhad and Hosseini-Hashemi (2014)

Table 3. Frequency ratios of the nanobeam for various mode numbers, amplitude ratios, nanobeam lengths, nonlocal parameters and boundary conditions

\begin{tabular}{|c|c|c|c|c|c|c|}
\hline \multirow{2}{*}{$D / r$} & \multirow{2}{*}{$\begin{array}{c}\text { Length } \\
{[\mathrm{nm}]}\end{array}$} & \multirow{2}{*}{$\begin{array}{c}\mu \\
{\left[\mathrm{nm}^{2}\right]}\end{array}$} & \multirow{2}{*}{$\begin{array}{l}\text { Boundary } \\
\text { condition }\end{array}$} & \multicolumn{3}{|c|}{ Mode number $n$} \\
\hline & & & & 1 & 2 & 3 \\
\hline \multirow{8}{*}{1} & \multirow{4}{*}{10} & \multirow{2}{*}{2} & SS & 0.9278 & 0.7921 & 0.6778 \\
\hline & & & $\mathrm{SC}$ & 0.9189 & 0.7864 & 0.6800 \\
\hline & & \multirow{2}{*}{4} & SS & 0.8724 & 0.6948 & 0.5832 \\
\hline & & & $\mathrm{SC}$ & 0.8595 & 0.6959 & 0.5982 \\
\hline & \multirow{4}{*}{20} & \multirow{2}{*}{2} & $\overline{\mathrm{SS}}$ & 0.9800 & 0.9278 & 0.8605 \\
\hline & & & $\mathrm{SC}$ & 0.9771 & 0.9233 & 0.8561 \\
\hline & & \multirow{2}{*}{4} & SS & 0.9613 & 0.8724 & 0.7764 \\
\hline & & & $\mathrm{SC}$ & 0.9561 & 0.8661 & 0.7728 \\
\hline \multirow{8}{*}{2} & \multirow{4}{*}{10} & \multirow{2}{*}{2} & SS & 0.9509 & 0.8621 & 0.7918 \\
\hline & & & $\mathrm{SC}$ & 0.9547 & 0.8751 & 0.8141 \\
\hline & & \multirow{2}{*}{4} & SS & 0.9141 & 0.8020 & 0.7377 \\
\hline & & & $\mathrm{SC}$ & 0.9239 & 0.8316 & 0.7806 \\
\hline & \multirow{4}{*}{20} & \multirow{2}{*}{2} & SS & 0.9863 & 0.9509 & 0.9062 \\
\hline & & & $\mathrm{SC}$ & 0.9869 & 0.9528 & 0.9100 \\
\hline & & \multirow{2}{*}{4} & SS & 0.9736 & 0.9141 & 0.8522 \\
\hline & & & $\mathrm{SC}$ & 0.9751 & 0.9190 & 0.8620 \\
\hline \multirow{8}{*}{3} & \multirow{4}{*}{10} & \multirow{2}{*}{2} & SS & 0.9677 & 0.9108 & 0.8674 \\
\hline & & & $\mathrm{SC}$ & 0.9867 & 0.9423 & 0.9069 \\
\hline & & \multirow{2}{*}{4} & SS & 0.9439 & 0.8736 & 0.8352 \\
\hline & & & $\mathrm{SC}$ & 0.9790 & 0.9281 & 0.8990 \\
\hline & \multirow{4}{*}{20} & \multirow{2}{*}{2} & SS & 0.9909 & 0.9677 & 0.9388 \\
\hline & & & $\mathrm{SC}$ & 0.9960 & 0.9768 & 0.9513 \\
\hline & & \multirow{2}{*}{4} & SS & 0.9826 & 0.9439 & 0.9046 \\
\hline & & & $\mathrm{SC}$ & 0.9925 & 0.9610 & 0.9274 \\
\hline
\end{tabular}


The above discussion verified the merit, high accuracy and applicability of the present exact solution, elliptic integrals, for nonlocal nonlinear free vibration analysis of nanobeams with various boundary conditions. In the following discussion, the small scale effects on the nonlinear free vibration of nanobeams are considered for higher mode numbers and phase plane diagrams $(\dot{q}(t)$ versus $q(t))$. A nanobeam made of aluminum (Al) with the bulk elastic properties $E=70 \mathrm{GPa}, \nu=0.35, \rho=2700 \mathrm{~kg} / \mathrm{m}^{3}$ (Ogata et al., 2002) and square cross section $b=2 h=0.1 L$ is considered as an illustrative example.
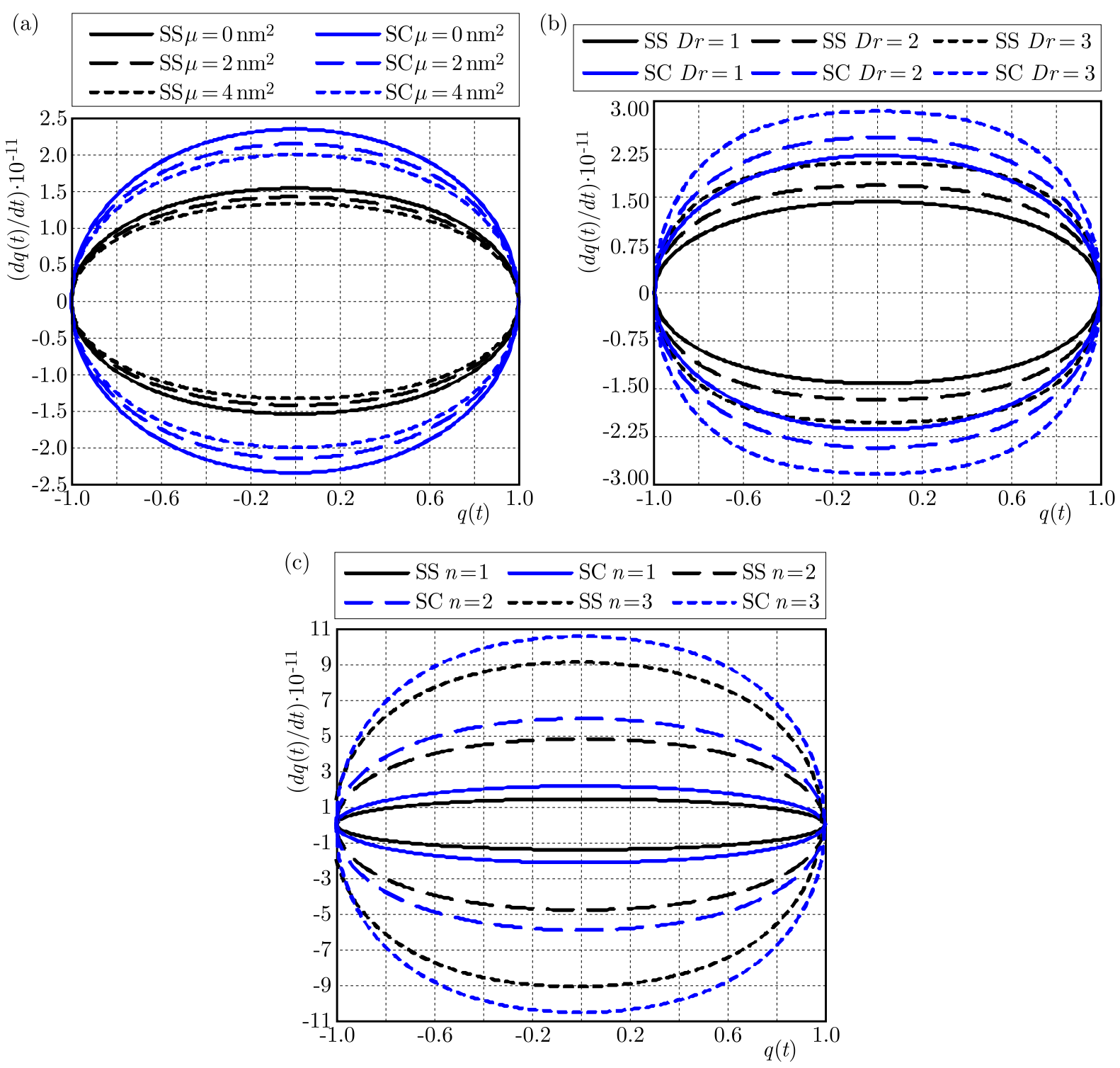

Fig. 2. Phase plane diagrams for the nanobeam: (a) $D / r=1, n=1, L=10 \mathrm{~nm}$; (b) $n=1, L=10 \mathrm{~nm}$, $\mu=2 \mathrm{~nm}^{2}$, (c) $D / r=1, L=10, \mu=2 \mathrm{~nm}^{2}$

Table 3 examines the small scale effects on the first three nonlocal to local nonlinear frequency ratios $\omega_{n l}^{n l} / \omega_{n l}$ of the SS and SC nanobeams for various amplitude ratios and nanobeam lengths. It is seen from Table 3 that the decreasing effect of the nonlocal parameter on the frequency ratios is greater at higher mode numbers. In addition, regardless of the mode number, by increasing the nanobeam length and amplitude ratio, the decreasing effect of the nonlocal parameter on the frequency ratio decreases. Moreover, except for $D / r=1$, the decreasing effect of the nonlocal parameter on the frequency ratio is more pronounced for nanobeams with softer boundary 
conditions. When the amplitude ratio equals to 1, similar effects can only be seen at higher mode numbers and small lengths.

Figures 2a-2c display the phase plane diagrams $(\dot{q}(t)$ versus $q(t))$ for nanobeams with different conditions. It is observed from Figs. 2a-2c that the phase plane diagrams of nanobeams in the presence of the small scale effect are symmetric ellipses, and the diagrams of SC nanobeams are similar to those of SS nanobeams. Figure 2a exhibits that consideration of the small scale effect decreases the area of the diagram, and this reduction becomes greater by increasing the value of the nonlocal parameter. But it is seen from Figs. $2 \mathrm{~b}$ and $2 \mathrm{c}$ that the area of the diagrams increases as the mode number and the amplitude ratio increase. In other words, an increase/decrease in the area of the diagrams due to the mentioned parameters is equivalent to an increase/decrease in the initial velocity condition.

\section{Conclusion}

This paper deals with exact solutions for large amplitude flexural vibration analysis of nonlocal Euler-Bernoulli nanobeams with SS and SC end conditions and von Kármán geometric nonlinearity. The equation of motion of nanobeam has been obtained in a nonlocal form and the exact analytical solution for natural frequency has been established using the exact elliptic integrals method. Two test examples are presented to demonstrate the accuracy of the formulation and applicability of the elliptic integrals method for nonlocal nonlinear free vibration analysis of nanobeams. It is observed that the small scale effect on the frequency ratios not only depends on the nanobeam dimensions and vibration amplitude, but also depends on the mode number and boundary conditions. In addition, it is seen that the small scale effect caused a reduction in the area of the phase plane diagrams of the nanobeams.

\section{References}

1. Ansari R., Ramezannezhad H., 2011, Nonlocal Timoshenko beam model for the large-amplitude vibrations of embedded multiwalled carbon nanotubes including thermal effects, Physica E, 43, $1171-1178$

2. Azrar L., Benamar R., White R., 1999, Semi-analytical approach to the non-linear dynamic response problem of S-S and C-C beams at large vibration amplitudes. Part I: General theory and application to the single mode approach to free and forced vibration analysis, Journal of Sound and Vibration, 224, 183-207

3. Azrar L., Benamar R., White R., 2002, A semi-analytical approach to the non-linear dynamic response problem of beams at large vibration amplitudes. Part II: Multimode approach to the steady state forced periodic response, Journal of Sound and Vibration, 255, 1-41

4. Bhashyam G., Prathap G., 1980, Galerkin finite element method for non-linear beam vibrations, Journal of Sound and Vibration, 72, 191-203

5. Byrd P.F., Friedman M.D., Byrd P., 1971, Handbook of Elliptic Integrals for Engineers and Scientists, Springer Berlin

6. Chandra R., Raju B.B., 1975, Large deflection vibration of angle ply laminated plates, Journal of Sound and Vibration, 40, 393-408

7. Chong A., Yang F., Lam D., Tong P., 2001, Torsion and bending of micron-scaled structures, Journal of Materials Research, 16, 1052-1058

8. Eringen A.C., 2002, Nonlocal Continuum Field Theories, Springer

9. Evensen D., 1968, Nonlinear vibrations of beams with various boundary conditions, AIAA Journal, 6, 370-372 
10. Fang B., Zhen Y.-X., Zhang C.-P., TAng Y., 2013, Nonlinear vibration analysis of doublewalled carbon nanotubes based on nonlocal elasticity theory, Applied Mathematical Modelling, 37, 1096-1107

11. Fleck N., Muller G., Ashby M., Hutchinson J., 1994, Strain gradient plasticity: theory and experiment, Acta Metallurgica et Materialia, 42, 475-487

12. Ghorbanpour Arani A., Atabakhshian V., Loghman A., Shajari A., Amir S., 2012 , Nonlinear vibration of embedded SWBNNTs based on nonlocal Timoshenko beam theory using DQ method, Physica B, 407, 2549-2555

13. Heireche H., Tounsi A., Benzair A., Manchou M., Adda Bedia E., 2008, Sound wave propagation in single-walled carbon nanotubes using nonlocal elasticity, Physica E, 40, 2791-2799

14. Hosseini-Hashemi S., Fakher M., Nazemnezhad R., 2013, Surface effects on free vibration analysis of nanobeams using nonlocal elasticity: A comparison between Euler-Bernoulli and Timoshenko, Journal of Solid Mechanics, 5, 290-304

15. Hosseini-Hashemi S., Nazemnezhad R., Bedroud M., 2014, Surface effects on nonlinear free vibration of functionally graded nanobeams using nonlocal elasticity, Applied Mathematical Modelling, 38, 3538-3553

16. Ke L.-L., Yang J., Kitipornchai S., 2010, An analytical study on the nonlinear vibration of functionally graded beams, Meccanica, 45, 743-752

17. Ke L., Xiang Y., Yang J., Kitipornchai S., 2009, Nonlinear free vibration of embedded doublewalled carbon nanotubes based on nonlocal Timoshenko beam theory, Computational Materials Science, 47, 409-417

18. Kiani K., 2010, Free longitudinal vibration of tapered nanowires in the context of nonlocal continuum theory via a perturbation technique, Physica E, 43, 387-397

19. Lestari W., Hanagud S., 2001, Nonlinear vibration of buckled beams: some exact solutions, International Journal of Solids and Structures, 38, 4741-4757

20. Ma Q., Clarke D.R., 1995, Size dependent hardness of silver single crystals, Journal of Materials Research, 10, 853-863

21. Malekzadeh P., Shojaee M., 2013, Surface and nonlocal effects on the nonlinear free vibration of non-uniform nanobeams, Composites, Part B - Engineering, 52, 84-92

22. Narendar S., Gopalakrishnan S., 2009, Nonlocal scale effects on wave propagation in multiwalled carbon nanotubes, Computational Materials Science, 47, 526-538

23. Nazemnezhad R., Hosseini-Hashemi S., 2014, Nonlocal nonlinear free vibration of functionally graded nanobeams, Composites Structures, 110, 192-199

24. Ogata S., Li J., Yip S., 2002, Ideal pure shear strength of aluminum and copper, Science, 298, $807-811$

25. Rao S.S., 2007, Vibration of Continuous Systems, John Wiley \& Sons

26. REDDy J., 2007, Nonlocal theories for bending, buckling and vibration of beams, International Journal of Engineering Science, 45, 288-307

27. REDDY J., 2010, Nonlocal nonlinear formulations for bending of classical and shear deformation theories of beams and plates, International Journal of Engineering Science, 48, 1507-1518

28. Setoodeh A., Khosrownejad M., Malekzadeh P., 2011, Exact nonlocal solution for postbuckling of single-walled carbon nanotubes, Physica E, 43, 1730-1737

29. Wang L., Hu H., 2005, Flexural wave propagation in single-walled carbon nanotubes, Physical Review B, 71, 195412

30. WANG Q., 2005, Wave propagation in carbon nanotubes via nonlocal continuum mechanics, Journal of Applied Physics, 98, 124301 
31. Wang Q., Varadan V., 2007, Application of nonlocal elastic shell theory in wave propagation analysis of carbon nanotubes, Smart Materials and Structures, 16, 178

32. Xie G., Han X., Liu G., Long S., 2006, Effect of small size-scale on the radial buckling pressure of a simply supported multi-walled carbon nanotube, Smart Materials and Structures, 15, 1143

33. Yang J., Ke L., Kitipornchai S., 2010, Nonlinear free vibration of single-walled carbon nanotubes using nonlocal Timoshenko beam theory, Physica E, 42, 1727-1735

34. YANG X., Lim C., 2009, Nonlinear vibrations of nano-beams accounting for nonlocal effect using a multiple scale method, Science China Technological Sciences, 52, 617-621

Manuscript received December 27, 2014; accepted for print December 18, 2016 